\title{
Trombosis carotidea en el lactante
}

DKES: GONZalo TORREAlba *. PATRICIO TAGLE *

Inrroducción. Los Accidentes Vasculares Enfálicos (A. V. E.) oclusivos del adulto tienen una frecuencia alta, habiéndose discutido extensamente su etiopatogenia, cuadro clínico y tratamiento. Conocido es también el papel que juega en ellos la oclusión (total o parcial) de la arteria carótida a nivel del cuello. (7-8).

Problenia aparte, lo constituyer los A. V. E. oclusivos en el niño, los cuales son poco conocidos y estudiados, quedando a menudo la etiología poco clara.

Además de la escasa frecuencia con que se describen estos casos, más excepcional aún resulta el compromiso de la arteria carótida a nivel del cuello, a diferencia del adulto.

Nuestro propósito es el presentar a un lactante, con una trombosis de carótida cervical y extendernos en algunas consideraciones a propósito de la etiología de estas oclusiones, considerando que no hernos encontrado referencias nacionales sobre el tema.

Caso clínico. Lactante de 1 año 5 meses da edad, nacida de parto eutócico a los $7 \frac{1}{2}$ meses de gestación. Sin antecedentes mórbidos de importancia, salvo un TEC con breve periodo de inconciencia al año de edad.

Consulta en un Policlínico periférico por presentar desde hacín tres días fiebre que llega hasta $40 \% \mathrm{C}$ y vómitos. Referido a otro Hospital, se constata al examęn de ingreso, una lactante eutrófica, bien hidratada, fabril (38\% C), decaída, palidez acentuada de piel y mucosa, siendo el examen segmentario normal. No había signos meníngeos. Se plantean los diagnósticos de infección urinaria y sepsis confirmándose el primero; días más tarde, por un urocultivo que revel6 desarrollo de E. Coli, e iniciándose la terapia con el antibiótico correspondiente (gentamicina).

\footnotetext{
* Unidael de Neurología. Facultad de Medicina, Universidad Católica de Chile. Santiago de Chile.
}

Permaneve febril, decaida e irritable, con compromiso del estado general y con acentuada palidez de piel y mucosa. Cin hemograma reveló anemia con 9,4 grs. Hb \% y Hcto. $31 \%$. Una punción lumbar dio salida a LCR claro cuyo cultivo fue negativo $y$ el examen citoquímico de coracteres normales.

Continúa en estas condiciones, reapareciendo vómitos y después de 11 días de hospitalizada, se instala en forma brusca una hemiparesia derecha hipotónica faciobraquio-crural, asociada a ptosis palpebral discreta $y$ miosis del ojo izquierdo. Había hipotreflexiá tendínea en extremidades derechas con el signo de Babruski a ese lado. No había signos meníngeos y el fondo de ojo era normal. Una nueva punción lumbar, dio salida a LCR cuyo cultivo fue negativo y citoquímico normal.

El cuadro neurológico no varió mayomente en los días siguientes, sin embargo, bajó la fiebre dos días más tarde, mejorando el estado general en forma progresiva. Sz hizo un estudio radiológico de cráneo y tórax, siendo ambos normales.

Vista $11 / 2$ mes más tarde en nuestro hospital, se comprueba hemiparesia derecha facio-braquio-crural en franca recuperación. Había desaparecido la ptosis palpebral y miosis izquierda, el examen físico general era negativo y babía un adecuado aumento pondo-estatural. Se practica una punción lumbar, que da salida a LCR claro, citoquímico normal. Un EEG. reveló un trazado francamente asimétrico con gran pobreza de ritmos rápidos sobre todas las áreas del hemisferio izquierdo. Se practica angiografía carotídea izquierda (Fig. 1) demostrándose oclusión total de carótida interna extracraneana a nivel de bifurcación đe carótida común, observándose sólo lene de carótida externa. Concordante con lo anterior, la cintigrafía cerebral (Fig. 2), reveló franco aumento del centelleo en hemisferio izquierdo. Por último una neumoencefalografía demostró una atrofia marcada de hemisferio izquíerdo. 


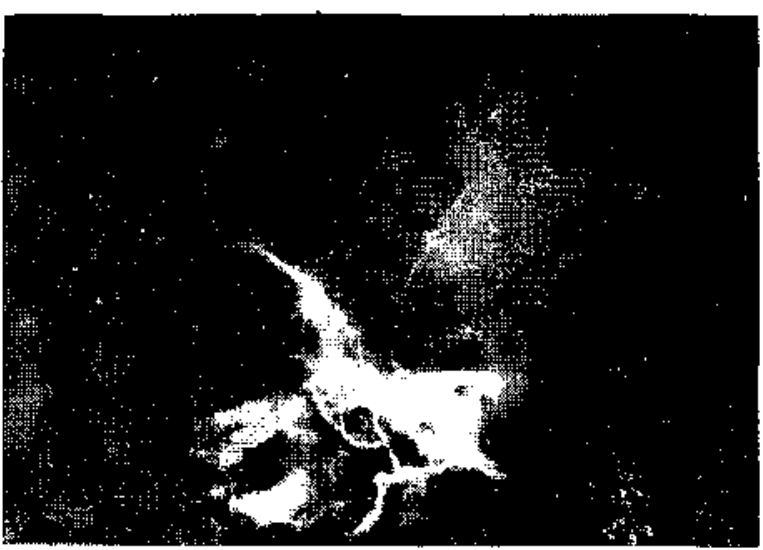

Figura Nọ 1. Anciogmfía Carotidea lzquicrda, Se aprecia oclusión de arteria carílicla interna cervical, sólo hay llente de ramas de carólida extema.

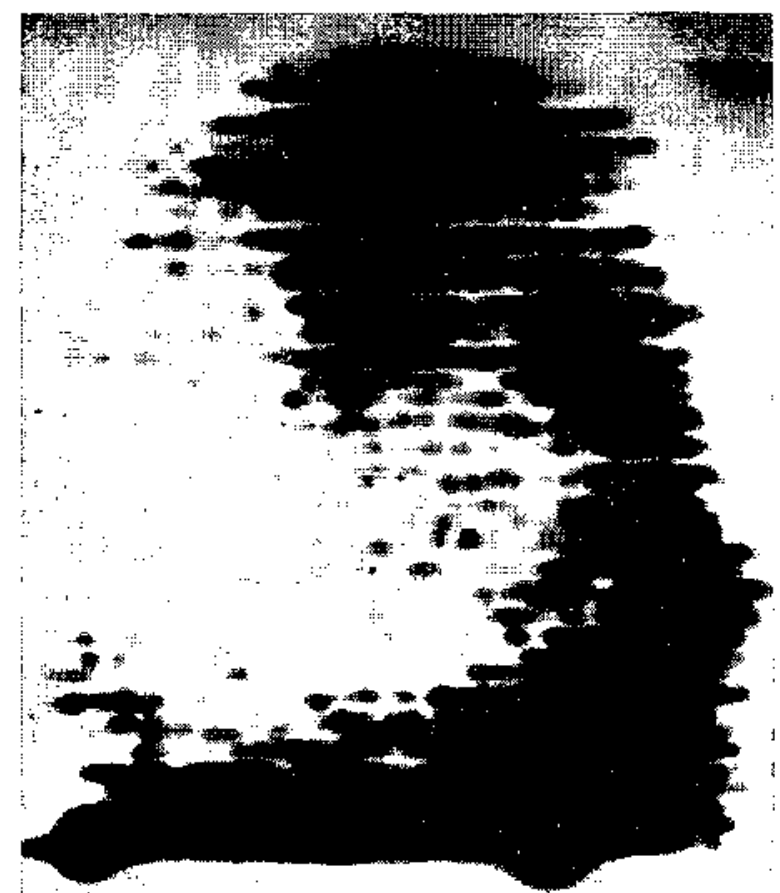

Figura N: 2. Cintigrafia ccrebral. Marcado aumento del centejleo en hemjsicrio izquielda, e proyección A-P.

Comentario. Durante la última década los A. V. E. oclusivos, en el niño, se diagnostican con más frecuencia, por $\mathrm{cl}$ uso de cada día mayor de la angiografía. Así, el térmito "Hemiplejia Infantil", que gencralmente se aplicaba a trombosis de scnos venosos o de venas corticales y a hemorragias, pasa a tener una nueva e importante causa en las trombosis arteriales cerebrales.
En cuarto a frecuencia podemos señalar que, Banker (2) en 1961 encuentra, 6 casos de trombosis arteriales cerebrales, en un estudio de 555 necropsias y sólo uno de ellos correspondia a trombosis carotídca. Por su parte, Wisoff (12), hace una revisión de la literatura en 1961, encontrando 27 casos de trombosis cerebrales en niños, a los que agrega dos más. Shillito (11), aporta el mayor número de trombosis cerebrales documentadas arteriográficamente, con 20 casos, constatándose que la mayoría de ellos presentaban trombosis de vasos intracraneanos, sicndo poco frecuente al compromiso de carótida cervical. Cabe destacar que sólo había 4 lactantes en esta serie, dos de los cuales presentaban trombosis de carótida cervical y otros dos de cerebral media. Davie (4), en 1967, encuentra descritos en la literatura, sólo 20 casos de trombosis carotídea cervical, de los cuales tres eran lactantes.

La etiología de las trombosis cerebrales en niños, generalmente es difícil de establecer, a diferencia del adulto en el cual la arterioesclerosis es sin duda la causa más frecuente. Davie (4), enumera los siguientes posibilidades etiológicas, en A. V. E. oclusivos en niños:

\section{I) Embolies:}

a) Cardiopatías congénitas - EBSA - Enfermedad rcumática.

b) Sepsis - Neumonía - Abscesos pulmonares.

II) Trombosis:

a) Cambios estructurales de la arteria.

1) Calcificaciones y Arterioesclerosis.

2) Aneurismas disecantes.

3) Necrosis de la media.

b) Traumatismos.

c) Infecciones.

d) Idiopáticas.

Si bien es cierto que esta clasificación abarca un gran número de posibilidades etiológicas, no es menos cierto que ha sido hecha tomando en consideración estudios necrópsicos. En la práctica clínica, uno se enfrenta a menor número de etiologías suceptibles de confirmar clínicamente, como serían:

a) Tratmatismos. Varios son los casos publicados, de troumas en la región cervical como en el cráneo, tanto en niños (6) como en adultos (9). En especial, los traumatismos de la faringe y re- 
gión amigdaliana que por su proximidad a la carótida, pueden complicarse más fácilmente con la obstrucción de este vaso.

b) Cardiopatias Congénitas. Los pacientes portadores de cardiopatias congénitas, están predispuestos a este tipo de A. V. E., en especial las formas con cianosis. Así, entre un 10-15\% de los niños que fallecen con cardiopatías cianóticas, se cncuentra en la nccropsia trombosis cerebrales y entre un $5-7 \%$ de los no cianóticos (1). Oclirren estos episodios en los dos primeros años de vida.

c) Compromiso directo del vaso, como en infecciones adyacentes, ya sean abcesos retrofaríngeos o periamidalianos, adenitis o bien tumores cerviciles. Explicables si tomamos en consideración la estrecha relación de la fosa amigdaliana y la entrada al canal carotídeo, pudiendo entonces un proceso inflamatorio extenderse a la adventicia del vaso, provocando una arteritis localizada (3).

De este modo el rubro de las idiopáticas adquierc relieve. Basta con afirmar que Davie (4), en sus 20 casos, concluye que "la etiología de la trombosis carotidea en ninguno de estos casos, se pudo comprobar fehacientemente" para dar una idea de la dificultad clínica para definir la etiología. De tal manera que en gran número de casos no habiendo una etiología clara para explicar la trombosis y teniendo como cuadro precedente una infección respiratoria alta, una otitis o sinusitis, se ha postulado que dada la proximidad de la carótida a estos procesos, podrían extenderse, probablemente vía linfática hacia carótida provocando una arteritis secundaria. (3-11). Así Shillito (11) encuentra que 18 de 25 casos, presentaban o habían presentado una infección respiratoria alta al producirse la trombosis cerebral. Por otra parte algunos (5) ban sostenido que cualquiera enfermedad con grave compromiso del estado general, podría producir desde un daño endotelial hasta necrosis de la pared arterial y consecuentementc una trombosis. Es así como esta complicación neurológica, puede presentarse aunque con poca frecuencia en estados sépticos y en otras infecciones graves. Un buen ejemplo es nuestro paciente, en que luego de casi dos semanas de un cuadro febril con grave compromiso del estado general, desarrolla bruscamente una hemiparesia derecha con un sindrome de Bernard Horner izquierdo, comprobánduse arteriográficamente una obstrucción carotídca. Habiéndose descartado una posible fuente de émbolos clínicamente y no habiendo evidencias de embolías en otros brganos, creemos que la etiopatogenia de esta trombosis carotídea puede catalogarse como una probable arteritis secundaria, a un proceso infeccioso grave, con daño de la pared del vaso, probablemente causado por microembolías con obstrucción de la vasa vasorum de la arteria, aunque no tenemos manera de comprobarlo. Descartamos a su vez el papel probable del TEC, por su lejanía al episodio de trombosis, ya que se describe esta causa como factor etiológico, hasta $36 \mathrm{hrs}$. después del traumatismo (6).

Por último, debemos recordar que el cuadro clínico al igual que en el adulto, se presenta en forma brusca, como en nuestro caso, en ocasiones asociado a convulsiones, o bien por períodos breves en que aparecen signos focales sensitivos o motores, que regresan hasta llegar a la trombosis definitiva del vaso (10). Es importante la detección en etapa precoz para su eventual trata. miento. Por lo cual, se debe tener presente que cuando un niño, que en el curso de una enfermedad infecciosa grave o de una infección respiratoria alta, desarrolla un cuadro neurológico como el descrito, se puede estar frente a una trombosis cerebral.

\section{RESUMEN}

Se presenta a un lactante de 1 año 5 meses de edad que en el curso de un cuadro infeccioso grave desarrolla una hemiparesia derecha con un sindrome de Bernard Horner izquierdo, certificándose posteriormente una trombosis carotídea a nivel cervical.

Se discuien algunos aspectos relacionados con la etiopatogenia de las trombosis cerebrales en el niño, y se plantea una posibilidad etiológica para el caso en estudio.

\section{REFERENCIAS}

1.- Aiata, J. Systemic and non arterioesclerotic causes of cerebral infections. Handbook of Clinical Neurology. Vascular Disease of the Nervous System. Pág. 447. Edit. North Holland Publishing Co. Germany, 1972.

2.-Banker, B. Cerebral vascular disease in infancy and thildhood. J. Neuropath. exp. Neurol. $20 \div 127$, 1960.

3.-Bickerstaff, E. Aetiology of acute hemiplegia in childhood. Brit. Med. J. 2: 82, 1964.

4.-Davie, J., Coke, W. Oclusive disease of carotid artery in children. Arch. Neurol. 17: 313, 1967.

5.-Ford, F,; Schaffer, A. (1927). Citado por Bickerstaff (3).

6.-Frantzen E.; Jacobsen, H. et al. Cerebral artery occlusion in children due to trauma to head and neck. Neurol. 11: 695, 1961. 
7.-Gurdjain, E.; Linder, D. et al. Cerebrovascular dìscase. An analysis of 600 cases. Neurol. 10: 372, 1960.

8.- Mutchinsen, $F$, Yates, $r$. Carotico-vertcbral stero. sis. Lancet 1: 2, 1957.

9.-Olaison, R.; Chistoferson, $L$. The syndrome of carotid occlusion following minor craniocerebral trau miti. 1. Neuro.t1rg. 3.3: 6.36, 1970.
10,- Stevens, H. Carotid artery occlusion in childhood Pediatrics 23: 699, 1959.

11,- Shillito, J. Catotid arteritis: A cause of homiplegin іл childhood. J. Neurosurg 21: 540, 1964.

12.-Wisolf. H.: Rothbatten, A. Cerebral artery thrombosis in children: Review of the literature and addition of two culses in apparently healthy children. Are. Neurol. 4: 258, 1961. 